\title{
Un espace pour chacun... Quand les expertises dialoguent
} Mention honorifique 2011

\section{A Place for Everyone . . . when Experts Speak With Each Other Un espacio para cada uno... Cuando las experiencias dialogan}

\author{
Silvie Delorme, Jacques Bélanger et Robert Desmeules
}

Volume 60, numéro 2-3, avril-septembre 2014

Bibliothèques et architecture

URI : https://id.erudit.org/iderudit/1025512ar

DOI : https://doi.org/10.7202/1025512ar

Aller au sommaire du numéro

Éditeur(s)

Association pour l'avancement des sciences et des techniques de la documentation (ASTED)

ISSN

0315-2340 (imprimé)

2291-8949 (numérique)

Découvrir la revue

Citer cet article

Delorme, S., Bélanger, J. \& Desmeules, R. (2014). Un espace pour chacun...

Quand les expertises dialoguent : mention honorifique 2011. Documentation et bibliothèques, 60(2-3), 79-84. https://doi.org/10.7202/1025512ar
Résumé de l'article

La Bibliothèque de l'Université Laval à Québec a mené, en 2009, un important projet de réaménagement et de reconceptualisation des services de la Bibliothèque des sciences humaines et sociales au Pavillon Jean-Charles-Bonenfant. Les trois articles qui suivent proposent trois visions différentes des membres de l'équipe du projet : deux représentants du client (Université Laval), soit la directrice de la bibliothèque et le directeur du Service des immeubles, ainsi que l'architecte consultant chargé de projet. Dialogue et vision partagée au menu.
Tous droits réservés (C) Association pour l'avancement des sciences et des techniques de la documentation (ASTED), 2014
Ce document est protégé par la loi sur le droit d'auteur. L'utilisation des services d’Érudit (y compris la reproduction) est assujettie à sa politique d'utilisation que vous pouvez consulter en ligne. 


\section{Un espace pour chacun... Quand les expertises dialoguent}

\section{SILVIE DELORME}

Consultante et directrice de la Bibliothèque de l'Université Laval de 2006 à 2012

silvie.delorme@videotron.ca

\section{RÉSUMÉ | ABSTRACT | RESUMEN}

La Bibliothèque de l'Université Laval à Québec a mené, en 2009, un important projet de réaménagement et de reconceptualisation des services de la Bibliothèque des sciences humaines et sociales au Pavillon Jean-Charles-Bonenfant. Les trois articles qui suivent proposent trois visions différentes des membres de l'équipe du projet: deux représentants du client (Université Laval), soit la directrice de la bibliothèque et le directeur du Service des immeubles, ainsi que l'architecte consultant chargé de projet. Dialogue et vision partagée au menu.

A Place for Everyone... when Experts Speak With Each Other

In 2009, the Library of the Université Laval in Québec City underwent an important remodelling and reconceptualisation of the services of the Humanities and Social Sciences Library, located in the JeanCharles-Bonenfant Building. The three articles that follow outline the different visions of three members of the project team: two representatives of the client (Université Laval), namely the library director and the director of building services, as well as the consulting architect and project manager. Dialogue and shared vision were at the heart of this project.

Un espacio para cada uno...

Cuando las experiencias dialogan

En 2009, la Biblioteca de la Universidad Laval en Quebec llevó a cabo un importante proyecto de reacondicionamiento $y$ de reconceptualización de los servicios de la Biblioteca de Ciencias Humanas y Sociales en el pabellón Jean-Charles-Bonenfant. Los siguientes tres artículos proponen tres visiones diferentes de los miembros del equipo de proyecto: dos representantes del cliente (la Universidad Laval), la directora de la biblioteca y el director del servicio inmobiliario, así como el arquitecto asesor a cargo del proyecto. Este artículo analiza el diálogo entre las partes y la visión compartida.

\section{Un espace savant métamorphosé!}

E N 2009, L'UNIVERSITÉ LAVAL RECEVAIT DU PROGRAMME des infrastructures du savoir du gouvernement fédéral une subvention de plus de huit millions de dollars pour débuter la rénovation de la Bibliothèque de l'Université Laval (BUL), soit le quatrième étage $\left(5000 \mathrm{~m}^{2}\right)$ de la Bibliothèque des sciences humaines et sociales $\left(22000 \mathrm{~m}^{2}\right)$, première étape d'un projet global de réaménagement de cette bibliothèque et de la Bibliothèque des sciences $\left(4200 \mathrm{~m}^{2}\right)$.

Inaugurée en 1969, la Bibliothèque des sciences humaines et sociales (BSHS) n'avait bénéficié d'aucun réaménagement important depuis. Conçue selon les critères et la vision de l'époque - un étage public avec comptoir de prêt et des étages de magasins pour les collections -, la bibliothèque nécessitait un réaménagement majeur afin de mieux répondre aux besoins des étudiants d'aujourd'hui. La réflexion du projet de réaménagement s'est déroulée sur plusieurs années : depuis un premier besoin, en 2004, de revoir l'organisation du quatrième étage où étaient logées les collections spéciales et de réunifier les multiples comptoirs de service jusqu'à la réflexion globale sur les espaces de la BUL qui se finalisera en 2010. Le concept retenu : un lieu de savoir et d'apprentissage, vivant, moderne et convivial, dont les espaces sont variés et lumineux.

\section{Un « parti bibliothéconomique » en amont des espaces à créer : une vision à partager}

Si le milieu de l'architecture parle de parti architectural pour un bâtiment, par analogie, la BUL a utilisé le terme de parti bibliothéconomique. Avant de se déployer dans l'espace, un projet architectural doit s'exprimer dans une vision claire et porteuse de sens, un concept fonctionnel qui répond aux besoins des usagers. C'est le rôle du bibliothécaire. Tout comme celui de partager, éduquer, expliquer et communiquer le projet.

Pour la Bibliothèque de l'Université Laval, il s'agissait de revoir son concept de service et de s'assurer que les espaces soient en concordance avec ce dernier. La BUL a d'abord choisi de mettre le lieu physique (et 


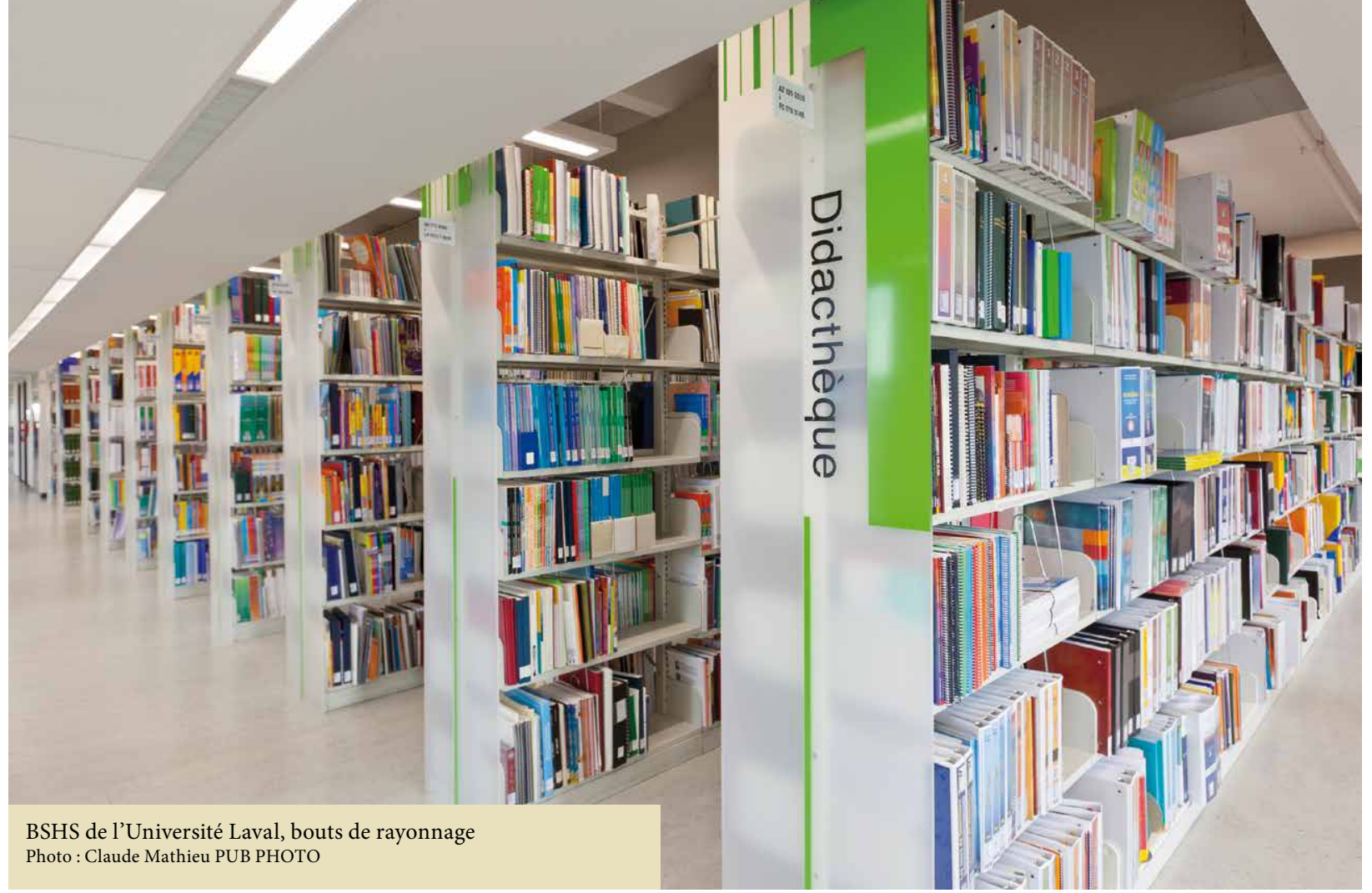

simultanément virtuel) au cœur du service et de l'accès aux collections par le biais de sa mission :

Infrastructure de recherche, lieu de savoir et d'apprentissage, la Bibliothèque de l'Université Laval offre et favorise l'accès au savoir à la communauté universitaire grâce à ses collections diversifiées, son personnel compétent, ses technologies performantes, ses locaux modernes et conviviaux et aux liens solides et féconds qu'elle tisse avec ses partenaires, tant internes qu'externes, et ses divers publics. ${ }^{1}$

Ensuite, il fallait qualifier le lieu et développer un concept de service clair et inclusif qui ancre les services dans cet espace à réaménager : lieu vivant de recherche, d'apprentissage et de médiation des savoirs; lieu central et ouvert où les espaces sont collaboratifs, modernes, adéquats et accueillants; espace offrant des collections riches, considérables et valorisées, et ayant un mandat de conservation du patrimoine documentaire universitaire; un service offert par un personnel qualifié et courtois; un niveau de service professionnel et personnalisé; un service appuyé par des technologies performantes.

Le projet, intégrant un agrandissement mineur en regard de la surface globale de la bibliothèque, devait rééquilibrer les espaces pour les usagers et ceux dévolus aux collections. La priorité est allée aux usagers. La bibliothèque a créé un Centre de conservation où sont logées les collections moins utilisées. Le choix de ces collections de conservation a été effectué en collaboration avec chacun des départements impliqués.

\footnotetext{
Delorme, Silvie. 2009. 40 ans plus tard... Une nouvelle bibliothèque de calibre international. Étude pour un projet de réaménagement des espaces de la Bibliothèque de l'Université Laval. Québec : Bibliothèque de l'Université Laval, 31.
}

\section{Des collections vers la connexion}

Les espaces devaient être repensés en fonction des nouvelles donnes sociales et technologiques : meilleure synergie collections/usagers; création de zones disciplinaires afin de favoriser le sentiment d'appartenance; regroupement des services et des ressources; places de travail nombreuses et variées; collections les plus utilisées et pertinentes en accès libre; espaces de conservation pour les collections semi-actives et fragiles; facilité d'accès aux documents sur demande; espaces pour étudiants diplômés redéployés pour une optimisation de l'utilisation, etc.

\section{Quand les expertises dialoguent}

À l'Université Laval, les projets architecturaux sont sous la responsabilité du Service des immeubles, qui travaille en étroite collaboration avec le service auteur

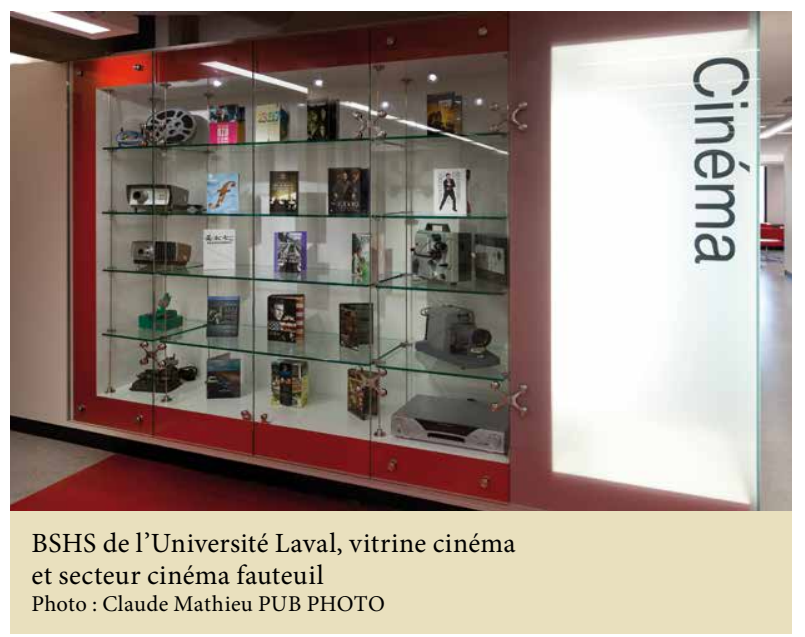




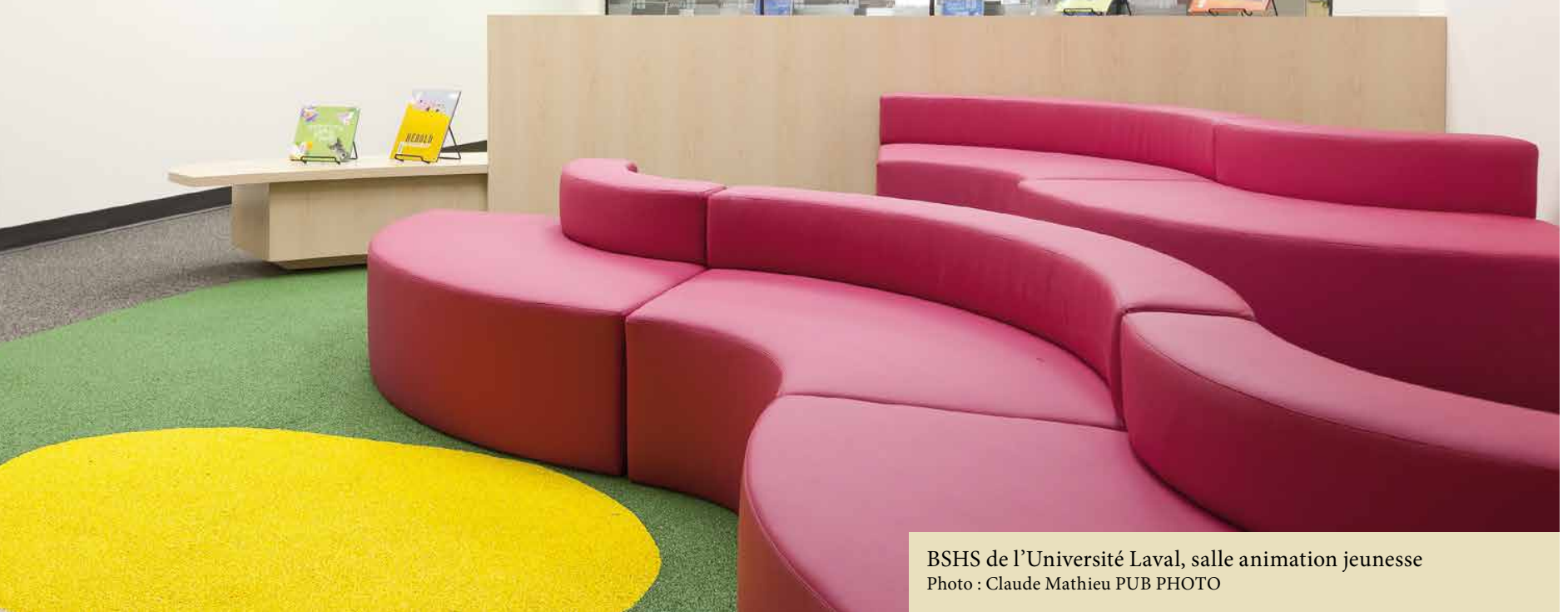

Les différents acteurs du projet ont donc dû cheminer en se ralliant à la nouvelle vision bibliothéconomique et en comprenant son impact sur l'aménagement des espaces et sur les coûts. Dans ce contexte, il était primordial que chacun des intervenants soit au même niveau et se sente autorisé d'apporter sa vision et son expertise spécifiques, et ce, dans le cadre de son propre mandat. Des présentations, des discussions animées et des visites ont permis d'articuler plus clairement le projet au moyen d'exemples et de mettre à niveau l'équipe de gestion intégrée du projet.

\section{Le bibliothécaire et l'architecte, se laisser surprendre...}

Le rôle du bibliothécaire, client du projet, consiste à identifier et à définir clairement ses besoins et les fonctionnalités de la bibliothèque. L'architecte crée, ancre dans la réalité cette idée exprimée. Il construit, dans tous les sens du terme. Il met en espace le projet bibliothéconomique et, pour ce faire, le repose sur un parti architectural. Il est essentiel de laisser la créativité s'exprimer en la stimulant, en permettant à l'architecte de nous surprendre et de nous proposer des solutions innovantes et inspirantes... dans le respect des budgets. La synergie bibliothécaire-architecte constitue le creuset dans lequel le projet prendra forme.

La dualité fond/forme, ou fonctionnalité/forme, est au cour de la création architecturale et les bâtiments les plus réussis le doivent certainement à un équilibre harmonieux entre ces deux besoins. Une forme créative marque le paysage urbain ou renouvelle entièrement l'aménagement d'un lieu. La forme architecturale idéale a une identité propre et forte, et permet à la fonction de rayonner et au service de remplir sa mission de manière optimale. Les deux éléments se renforcent l'un l'autre et ajoutent profondeur artistique et esthétique à un espace de vie où l'activité humaine se déploie. Le beau inspire et peut transporter l'humain en des lieux insoupçonnés. Larchitecture en est un pont.

L'écart entre les besoins fonctionnels et la créativité spatiale et architecturale émane généralement de l'attitude des uns et des autres soit à vouloir imposer leur vision, soit à leur incapacité à se positionner et à exprimer leurs besoins. L'architecte n'est pas l'ennemi du bibliothécaire, et vice-versa. Dans cette incompréhension se glisse souvent une mauvaise écoute de l'autre et de sa spécificité. La reconnaissance et le respect des compétences et des expertises des uns et des autres permettent de trouver l'angle d'équilibre, pivot d'un projet porteur et créatif. Certains architectes ne disent-ils pas que les meilleurs projets sont le fruit des clients les plus exigeants, les plus ouverts et visionnaires?

Euvrer ou participer à un projet d'architecture en compagnie d'architectes vifs d'esprit, créatifs et compétents est un bonheur réel, peu fréquent dans une vie, qui permet de matérialiser ce qui n'était à l'origine peut-être qu'une simple idée ou l'expression d'un besoin. Un pur geste créateur au bénéfice des bibliothèques et du savoir! 


\section{Le point de vue du directeur du Service des immeubles}

\section{ROBERT DESMEULES}

Directeur du Service des immeubles de l'Université Laval de 2006 à 2011 robert.desmeules58@gmail.com

Pour une bonne compréhension des enjeux du projet, il est important de rappeler que le Pavillon Jean-Charles-Bonenfant, construit en 1968, devait à l'origine être dédié entièrement à la fonction bibliothèque et loger uniquement la Bibliothèque de l'Université Laval (c'est-à-dire la Bibliothèque des sciences humaines et sociales (BSHS) et la Bibliothèque scientifique). Or, la communauté universitaire scientifique tenait à une bibliothèque à proximité des pavillons de sciences. Il avait donc été convenu de modifier le projet initial et de loger la BSHS au Pavillon Bonenfant et la Bibliothèque scientifique au Pavillon Vachon. Le Pavillon Bonenfant avait néanmoins été construit avec les mêmes dimensions qu'initialement prévues et, comme à cette époque, la BSHS n'était pas suffisamment grande pour occuper toute la superficie du nouveau pavillon, on avait planifié y installer diverses unités administratives pour compléter les espaces disponibles, et ce, dans une proportion assez importante de la superficie totale du bâtiment. Or, le rêve du Pavillon Jean-Charles-Bonenfant dédié uniquement à la bibliothèque a perduré, tout au long de ces années, tant dans l'imaginaire des employés de la bibliothèque que dans celui de plusieurs membres de la communauté universitaire.

À l'époque de la construction, les collections de la bibliothèque sur les étages n'étaient pas accessibles à la clientèle, et les systèmes mécaniques avaient été conçus pour des magasins de livres. Afin de répondre aux besoins qui évoluaient, plusieurs changements ont été apportés au fonctionnement de la bibliothèque au cours des 30 dernières années, et plusieurs scénarios ont été élaborés pour dégager plus d'espaces pour les collections, mais également pour les usagers. Le regroupement des deux bibliothèques a même été étudié à nouveau. Parallèlement, pendant cette période, le Service des immeubles a été saisi de plusieurs plaintes de la part des usagers quant à la qualité de l'air dans le pavillon.

\section{Le projet de réaménagement et les visions}

Lors de la réception de la subvention du gouvernement fédéral en 2009, le Service des immeubles considérait avant tout le nouveau projet de réaménagement de la Bibliothèque des sciences humaines et sociales comme un projet de maintien d'actifs (rénovation) et d'une mise aux normes étalée sur plusieurs phases. Il estimait que les budgets obtenus des instances gouvernementales devaient servir à cet effet.

La direction du Service des immeubles croyait bien comprendre les besoins et les contraintes de la bibliothèque et, inversement, a tenu pour acquis que la bibliothèque connaissait bien les contraintes du Service des immeubles. Un mandat a donc été confié à des firmes professionnelles en présumant que les contraintes et les besoins des deux clients étaient bien connus et partagés par les représentants des deux unités.

Or, lors de la présentation des premiers concepts, il est apparu clairement que les deux services avaient de grandes divergences sur les attentes et la finalité du projet. Les architectes ont accordé une très grande écoute à la vision de la bibliothèque, qui impliquait un nouveau concept global selon les documents remis à la direction de l'Université pour la demande de subvention. Plusieurs rencontres internes ont été requises entre le Service des immeubles et la bibliothèque afin de réajuster la compréhension mutuelle des besoins, ce qui a impliqué un certain délai dans l'échéancier afin que les ajustements aux besoins et au mandat soient apportés.

Les nombreuses rencontres ont permis d'apporter les compromis requis de part et d'autre afin de réaliser un projet extraordinaire pour les deux parties. La direction de l'Université Laval a été rencontrée pour valider les nouvelles orientations et les ajustements budgétaires qui découlaient de ces nouvelles orientations. Des démarches ont enfin été entreprises auprès des instances gouvernementales pour présenter les stratégies à long terme de ce projet de maintien d'actifs avec un réaménagement important des espaces de la bibliothèque. 